\title{
Asteroseismology with the WIRE satellite
}

\section{Combining ground- and space-based photometry of the $\delta$ Scuti star $\epsilon$ Cephei}

\author{
H. Bruntt ${ }^{1,2}$, J. C. Suárez ${ }^{3,4, \star}$, T. R. Bedding ${ }^{2}$, D. L. Buzasi ${ }^{5}$, A. Moya ${ }^{4}$, P. J. Amado ${ }^{6,3}$, S. Martín-Ruiz ${ }^{3}$, R. Garrido ${ }^{3}$, \\ P. López de $\mathrm{Coca}^{3}$, A. Rolland ${ }^{3}$, V. Costa ${ }^{3}$, I. Olivares ${ }^{3}$, and J. M. García-Pelayo ${ }^{3}$
}

${ }^{1}$ Niels Bohr Institute, Juliane Maries Vej 30, University of Copenhagen, Denmark

e-mail: bruntt@physics.usyd.edu.au

2 School of Physics A28, University of Sydney, 2006 NSW, Australia

e-mail: t. bedding@physics .usyd.edu.au

3 Instituto de Astrofísica de Andalucía, CSIC, CP3004 Granada, Spain

e-mail: jcsuarez@iaa.es

4 Observatoire de Paris, LESIA, UMR 8109, Meudon, France

e-mail: andy.moya@obspm.fr

5 US Air Force Academy, Department of Physics, CO, USA

e-mail: derek.buzasi@usafa.af.mil

${ }^{6}$ Universidad de Granada, Departamento Física Teórica y del Cosmos, Campus Fuentenueva, Granada, Spain

Received 7 June 2006 / Accepted 20 September 2006

\section{ABSTRACT}

\begin{abstract}
Aims. We have analysed ground-based multi-colour Strömgren photometry and single-filter photometry from the star tracker on the WIRE satellite of the $\delta$ Scuti star $\epsilon$ Cephei.

Methods. The ground-based data set consists of 16 nights of data collected over 164 days, while the satellite data are nearly continuous coverage of the star during 14 days. The spectral window and noise level of the satellite data are superior to the ground-based data and this data set is used to locate the frequencies. However, we can use the ground-based data to improve the accuracy of the frequencies due to the much longer time baseline.

Results. We detect 26 oscillation frequencies in the WIRE data set, but only some of these can be seen clearly in the ground-based data. We have used the multi-colour ground-based photometry to determine amplitude and phase differences in the Strömgren $b-y$ colour and the $y$ filter in an attempt to identify the radial degree of the oscillation frequencies. We conclude that the accuracies of the amplitudes and phases are not sufficient to constrain theoretical models of $\epsilon$ Cep. We find no evidence for rotational splitting or the large separation among the frequencies detected in the WIRE data set.

Conclusions. To be able to identify oscillation frequencies in $\delta$ Scuti stars with the method we have applied, it is crucial to obtain more complete coverage from multi-site campaigns with a long time baseline and in multiple filters. This is important when planning photometric and spectroscopic ground-based support for future satellite missions like COROT and KEPLER.
\end{abstract}

Key words. stars: oscillations - stars: variables: $\delta$ Sct - stars: individual: $\epsilon$ Cephei (HD 211336; HR 8494) - techniques: photometric

\section{Introduction}

$\delta$ Scuti stars are main sequence population I A- and F-type stars. They are found in the classical Cepheid instability strip on the main sequence, have masses around twice solar, and temperatures around $7500 \mathrm{~K}$. They have only very shallow outer convection zones but their cores are fully convective. Most stars found in the instability strip are variable and most are multi-periodic, with periods around $1-2 \mathrm{~h}$ and amplitudes typically at the mmag level, although some have amplitudes above 0.1 mag (Rodríguez et al. 2000).

To get a better understanding of $\delta$ Scuti stars, several multisite ground-based campaigns have carried out extensive monitoring of selected targets. For example, the Delta Scuti Network (Zima 1997; Zima et al. 2002) has monitored FG Virginis during several seasons and 79 frequencies have been detected (Breger et al. 2005). In that case, data from several ground-based observatories collected over 13 years were combined, which

^ Associate researcher at l'Observatoire de Paris. allowed the detection of frequencies down to amplitudes of just 0.2 mmag in Strömgren $y$. It is important to note that about 50 of the reported frequencies have amplitudes below $0.5 \mathrm{mmag}$. Breger et al. (2005) stated that typical multi-site campaigns with a duration of 200 to $300 \mathrm{~h}$ detect only $5-10$ frequencies. Since so few frequencies are detected, while theory predicts a much higher number of excited modes, Breger et al. (2005) concluded that either longer ground-based photometric campaigns or highprecision space-based campaigns are necessary.

Buzasi et al. (2005) recently found Altair to be a lowamplitude $\delta$ Scuti star based on high-precision photometry from the star tracker on the Wide-field InfraRed Explorer (WIRE) satellite. They found only seven frequencies but three of these had amplitudes around $0.1 \mathrm{mmag}$. The reason why so few frequencies were detected in Altair compared to FG Vir may be the much higher rotation rate of Altair, which has $v \sin i$ $=210 \pm 20 \mathrm{~km} \mathrm{~s}^{-1}$ while FG Vir has $v \sin i$ around $21 \mathrm{~km} \mathrm{~s}^{-1}$ (Mantegazza \& Poretti 2002; Mittermayer \& Weiss 2003). Suarez et al. 2005 have calculated models of Altair and used 
the constraints from the frequencies detected by Buzasi et al. (2005). Suarez et al. (2005) found that high rotational velocity makes the interpretation of the frequencies difficult, due to the limits of the applied second order perturbation theory and effects of near degeneracy. The $\delta$ Scuti star treated in this paper has $v \sin i \simeq 90 \mathrm{~km} \mathrm{~s}^{-1}$ and may present a simpler case. The studies of Breger et al. (2005) and Buzasi et al. (2005) both agree that high photometric precision and long temporal coverage is needed to fully explore the oscillation spectra of $\delta$ Scuti stars.

In the present study we have combined two quite different photometric data sets for the multi-mode $\delta$ Scuti star $\epsilon$ Cephei (HD 211336). One data set is from the WIRE satellite covering 14 days with very high signal-to-noise $(S / N)$. The other data set is single-site ground-based Strömgren $u v b y$ photometry from 16 nights collected during 164 days, thus having gaps from a single day to several weeks. We find that by combining the very different properties of the data sets in terms of $S / N$ and spectral window, we can measure the frequencies very accurately. We assess the uncertainties on the measured frequencies from extensive simulations to see if mode identification is possible using amplitude ratios and phase differences in the modes measured with the Strömgren filters.

\subsection{Previous studies of $\epsilon$ Cep}

$\epsilon$ Cep was first identified as a $\delta$ Scuti star by Breger (1966) who found a single frequency at $f=23.8 \pm 1.7 \mathrm{c} /$ day based on only two nights of data. One night of $y$ band photometry by Fesen (1973) showed an apparent change in amplitude, but today we know that this is likely due to beating of frequencies. Spectroscopy was first carried out during three nights by Gray (1971), who confirmed the period known from photometry. The mean radial velocity amplitude measured on the three nights was $15 \pm 3 \mathrm{~km} \mathrm{~s}^{-1}$, but this disagrees with upper limits of $\sim 1 \mathrm{~km} \mathrm{~s}^{-1}$ found by Kennelly et al. (1999) and Baade et al. (1993).

Line profile variations (LPVs) were detected by Baade et al. (1993). Based on only nine spectra they suggested that the observed LPV could be explained by a $p$ mode with high azimuthal order $m \sim 6-8$. More extensive monitoring with high-resolution spectroscopy was done by Kennelly et al. (1999). They monitored $\epsilon$ Cep in a multi-site campaign during eight nights. They detected a rich set of frequencies in the range 17-40 c/day with radial degrees $l=5-15$ using two-dimensional Fourier analysis, but their results were preliminary.

Costa et al. (2003) monitored $\epsilon$ Cep with Strömgren filters but they concluded that the rich amplitude spectrum could not be adequately resolved based on their single-site data set. We will use this data set in the present study.

\section{Observations}

\subsection{Observations from the ground}

$\epsilon$ Cephei (HD 211336) was monitored with simultaneous $u v b y$ measurements from Observatorio de Sierra Nevada (OSN) in 2001-2 on 16 nights during a period of 164 days (Costa et al. 2003). Eight nights of data were collected from 2001 August 9-25. There is data from October 8 and two nights on 2001 November 13 and 17. Four nights were obtained from 2001 December 3 to 9 . Finally, data from a single night was obtained on 2002 January 20.

$\epsilon$ Cep was observed for $6-8 \mathrm{~h}$ each night and a total of 2250 data points were collected. After removal of the oscillations the rms noise is $3.3 \mathrm{mmag}$ in $u$ and $1.8 \mathrm{mmag}$ in $v, b$ and $y$.
Table 1. Basic photometric indices for $\epsilon$ Cephei.

\begin{tabular}{rrrrrr}
\hline \hline$V$ & $B-V$ & $b-y$ & $m_{1}$ & $c_{1}$ & $\mathrm{H}_{\beta}$ \\
\hline 4.19 & 0.28 & $0.171(2)$ & $0.192(4)$ & $0.784(4)$ & 2.761 \\
\hline
\end{tabular}

The complete light curve is shown in the top panel in Fig. 1 while details of the stellar oscillations are seen in the bottom panel. The zero point in time is $t_{0}=2452130$.

\subsection{Observations from space}

The Wide-field InfraRed Explorer (WIRE) satellite mission was designed to study star-burst galaxies in the infrared (Hacking et al. 1999). Unfortunately, the hydrogen which was to be used for cooling the main camera was lost soon after launch. Since 1999 the $52 \mathrm{~mm}$ star tracker on WIRE has been used to monitor bright stars continuously for one to six weeks (see Bruntt \& Buzasi 2006).

$\epsilon$ Cep was observed with WIRE from 2004 June 20 to July 4. The raw data set consists of around $6000008 \times 8$ pixel CCD windows centered on the star with a time-sampling of $0.5 \mathrm{~s}$. The data were reduced as described by Bruntt et al. (2005) and points taken within $15 \mathrm{~s}$ were binned. The resulting light curve has 25293 data points collected during 13.6 days with three short gaps with durations of $0.2,0.2$, and 0.5 days. The complete light curve is shown in Fig. 1 where the zero point in time is $t_{0}=2453175.5$. Note that the WIRE observations started about 2.9 years after the OSN run.

The rms noise level in the WIRE data set after removal of the oscillations is $1.7 \mathrm{mmag}$. To estimate the white noise component we calculated the noise in the amplitude spectrum at high frequencies $(10 \pm 0.5 \mathrm{mHz}$; the Nyquist frequency is $33.3 \mathrm{mHz}$ ). From this we found the noise level to be $12.3 \mathrm{ppm}$ or $1.2 \mathrm{mmag}$ per $15 \mathrm{~s}$ bin. Each WIRE observation collects about $e_{\text {star }}=10^{5}$ electrons, and after binning every 30 data points (15 s sampling), the theoretical Poissonian noise is $0.6 \mathrm{mmag}$ or more than a factor two lower than the actual observed noise level. The higher noise level is due to the relatively high sky level during the $\epsilon$ Cep run. We estimate the noise contribution from the background to be $\sigma_{\mathrm{AP}}^{2}=n_{\text {pix }} *\left(e_{\text {sky }}+\sigma_{\mathrm{ro}}^{2}\right) / e_{\mathrm{star}}^{2}$ following Eq. (31) in Kjeldsen \& Frandsen (1992); here $n_{\text {pix }}$ is the number of pixels in the aperture, $\sigma_{\text {ro }}$ is the readout noise, while $e_{\text {star }}$ and $e_{\text {sky }}$ are the number of electrons from the star and sky background, respectively. We use $n_{\text {pix }}=12$, a gain of $15 e^{-1} / \mathrm{ADU}, e_{\mathrm{sky}}=420 \pm 220 \mathrm{ADU}$, and a $\sigma_{\mathrm{ro}}=10 e^{-1}$ to obtain $\sigma_{\mathrm{AP}}=2.7 \pm 0.7 \mathrm{mmag}$ which is comparable to the Poissionian noise $\sigma_{\text {count }}=1 / \sqrt{e_{\text {star }}}=3.2 \mathrm{mmag}$. This explains the relatively high noise level.

\section{Fundamental parameters of $\epsilon$ Cep}

The basic photometric indices for $\epsilon$ Cep are summarized in Table 1. The $V$ magnitude and $B-V$ colour are based on 12 measurements and are taken from Mermilliod's compilation of Eggen's $U B V$ data (available through SIMBAD). The Strömgren indices are from Hauck \& Mermilliod (1998) and are based on a combination of 60 measurements, while $\mathrm{H}_{\beta}$ is based on 47 measurements. The projected rotational velocity $(v \sin i)$ of $\epsilon$ Cep is $105 \mathrm{~km} \mathrm{~s}^{-1}$ according to 10 measurements from Bernacca \& Perniotto (1970), while Royer et al. (2002) found $91 \mathrm{~km} \mathrm{~s}^{-1}$. The typical uncertainty on $v \sin i$ is $5 \%$ (Royer et al. 2002), i.e. $\sigma(v \sin i)=5 \mathrm{~km} \mathrm{~s}^{-1}$. 


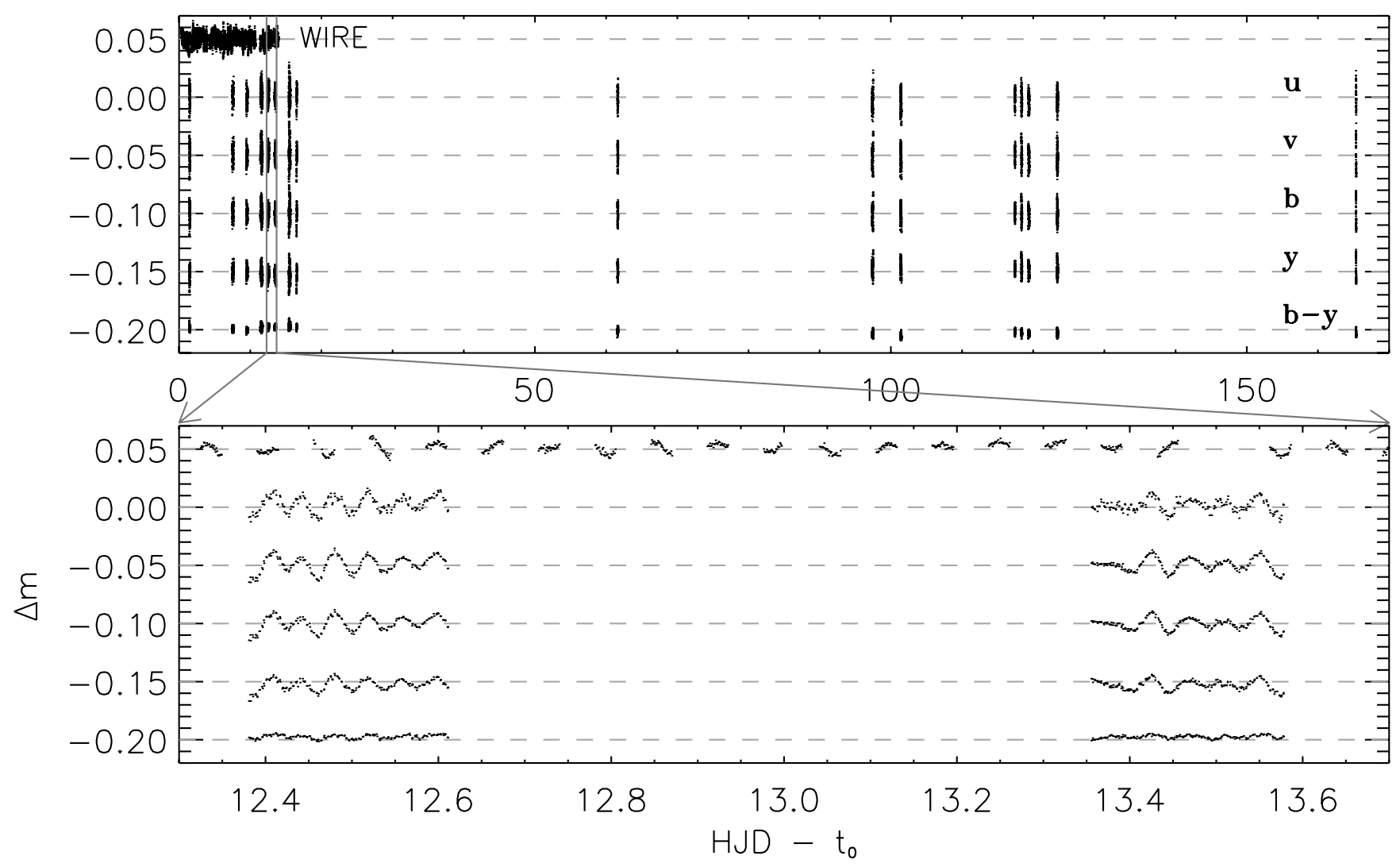

Fig. 1. Each panel shows the light curve of $\epsilon$ Cep from WIRE and OSN in the uvby filters and $b-y$. The top panel shows the complete set of light curves and the bottom panel shows the details of a small part. Note that the OSN data were collected about 2.9 years prior to the WIRE data, i.e. the zero points $t_{0}$ are different (see main text).

We used TEMPLOGG (Rogers 1995) to determine the fundamental atmospheric parameters of $\epsilon$ Cep and the results are $T_{\text {eff }}=7340 \pm 50, \log g=3.9 \pm 0.1,[\mathrm{Fe} / \mathrm{H}]=0.12 \pm 0.04$. This is consistent with the spectral type F0 IV. We stress that the quoted uncertainties are based solely on the uncertainty on the photometric indices. Realistic uncertainties on $T_{\text {eff }}, \log g$, and $[\mathrm{Fe} / \mathrm{H}]$ are $150 \mathrm{~K}, 0.2 \mathrm{dex}$, and 0.2 dex (Rogers 1995; Kupka \& Bruntt 2001). Based on the Strömgren $b-y$ and $\mathrm{H}_{\beta}$ indices there is no significant interstellar reddening.

Erspamer \& North $(2002,2003)$ used an automated procedure to determine individual abundances of 140 A- and F-type stars and $\epsilon$ Cep was included in their data set. They used Geneva photometry to fix $T_{\text {eff }}=7244 \pm 150 \mathrm{~K}$ and the HIPPARCos parallax and evolution models to find $\log g=4.02 \pm 0.2$, which both agree with our Strömgren photometry when using TEMPLOGG. The abundance analysis of $\epsilon$ Cep yielded $[\mathrm{Fe} / \mathrm{H}]=+0.08 \pm 0.10$, which is also in good agreement with TEMPLOGG. The error estimate on $[\mathrm{Fe} / \mathrm{H}]$ given here is based on the various contributions to the error budget, as discussed in detail by Erspamer \& North (2002).

The location of $\epsilon$ Cep in the Hertzsprung-Russell (HR) diagram is shown in Fig. 2. We show evolutionary tracks from Lejeune \& Schaerer (2001) for solar metallicity $(Z=0.02)$. The dashed track is for a metallicity of twice the solar value for a mass $M / M_{\odot}=2.0$. To estimate the luminosity, we used the visual magnitude $V=4.19 \pm 0.03$ and the HIPPARCOS parallax of $38.9 \pm 0.5$ mas (ESA 1997). We found the bolometric correction (BC) by interpolation in the tables by Bessell et al. (1998), i.e. $\mathrm{BC}=0.04 \pm 0.02$. For the solar bolometric magnitude we used $4.75 \pm 0.04$. Thus, we find $L / L_{\odot}=10.7 \pm 0.6$ and adopt $T_{\text {eff }}=7340 \pm 150 \mathrm{~K}$. The $1 \sigma$ error box is indicated in Fig. 2 .
From the location of $\epsilon$ Cep in the HR diagram relative to the evolutionary tracks from Lejeune \& Schaerer (2001), and adopting a metallicity $[\mathrm{Fe} / \mathrm{H}]=0.1 \pm 0.1$, we estimate the mass to be $M / M_{\odot}=1.75 \pm 0.20$.

From the estimated mass, temperature, and parallax we can calculate the surface gravity using $\log g_{\pi}=4\left[T_{\text {eff }}\right]+[M]+$ $2 \log \pi+0.4\left(V+B C_{V}+0.26\right)+4.44$, where $\left[T_{\text {eff }}\right]=\log \left(T_{\text {eff }} / T_{\text {eff } \odot}\right)$ and $[M]=\log \left(M / M_{\odot}\right)$. For $\epsilon$ Cep we find $\log g_{\pi}=4.0 \pm 0.1$ which agrees with Erspamer \& North (2003) and the photometric calibration from TEMPLOGG.

\section{Time series analysis}

\subsection{Spectral windows}

To illustrate the difference between the WIRE and OSN data sets in the frequency domain we have calculated the spectral windows. To do this we used the same observation times as in the real data sets and inserted an artificial sinusoidal signal at $f=20 \mathrm{c} /$ day. The resulting spectral windows for the WIRE and OSN data sets are shown in Fig. 3 in the top and bottom panels, respectively.

The complexity of the OSN spectral window is apparent, with several alias peaks at $1.0,0.5$, and $0.01 \mathrm{c} / \mathrm{d}$. The latter is seen in the inset in Fig. 3 and arises from the large gaps in the osN time series, i.e. $f \simeq 1 / T_{\text {obs }} \simeq 0.01 \mathrm{c} /$ day, since the total observing time is $T_{\mathrm{obs}}=122$ days. Note that we decided not to use the last night from OSN in the analysis since it degrades the spectral window. The reason is the long gap of 42 nights from night 15 to night 16. 


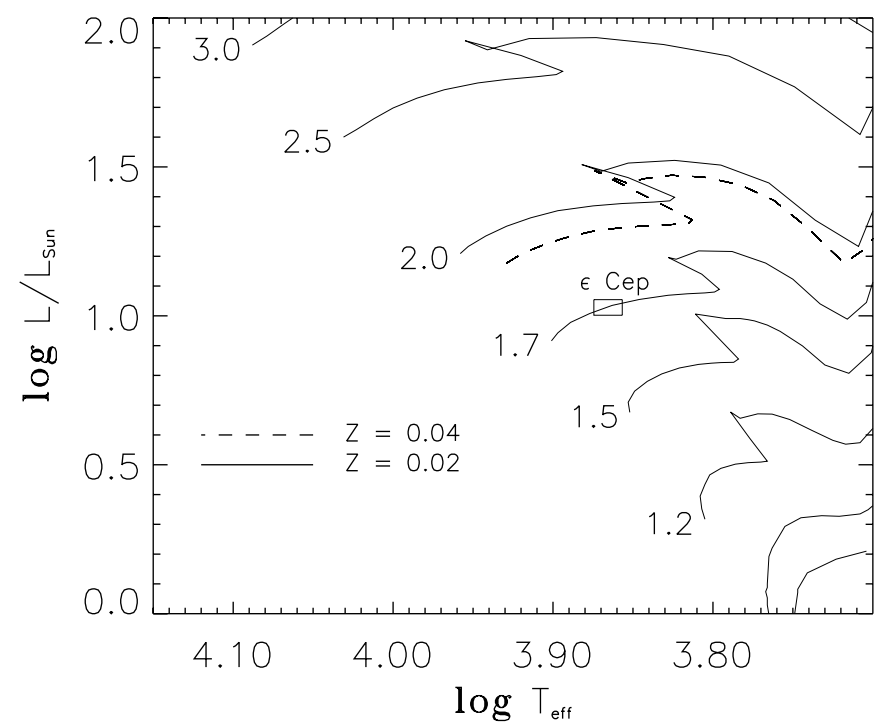

Fig. 2. HR diagram with evolution tracks from Lejeune \& Schaerer (2001) for $Z=0.02$ (solar) and $Z=0.04$. The $1 \sigma$ error box for $\epsilon$ Cep is indicated and is consistent with a slightly evolved star with mass $M / M_{\odot}=1.75 \pm 0.20$ for a metallicity of $[\mathrm{Fe} / \mathrm{H}]=0.1$.

As a result of the long gaps in the OSN time series, the peaks in the amplitude spectrum at $f \pm n \times 1 / T_{\text {obs }}$ - where $n$ is an integer - are almost equally good solutions. However, if one can be sure about selecting the "right peak," the accuracy of the frequency is significantly better than in the WIRE data set. This may prove difficult since in the real data set the spectrum is affected by closely spaced frequencies and noise sources such as photon shot noise and non-white instrumental drift noise.

The WIRE spectral window has a much more well-defined peak. During each WIRE orbit the satellite switches between two targets in order to minimize the effect of scattered light from the illuminated face of the Earth. Thus, $\epsilon$ Cep was observed with a duty cycle of $\simeq 40 \%$ (cf. bottom panel in Fig. 1 ). As a consequence, significant alias peaks are seen at frequencies that are combinations of the frequency of the oscillation signal and the orbital frequency of WIRE, $f_{i}=\left|f \pm n f_{\mathrm{W}}\right|$, where $f$ is the genuine frequency, $n$ is an integer, and $f_{\mathrm{W}}$ is the orbital frequency: $f_{\mathrm{W}}=15.348 \pm 0.001 \mathrm{c} /$ day. The first set of side lobes have amplitudes relative to the main peak of $76 \%$.

\subsection{Observed amplitude spectra}

The observed amplitude spectra are shown in Fig. 4. In the two top panels we have used the WIRE data set: the first panel is an overview and the second panel shows the details of the region $12-28 \mathrm{c} /$ day where the oscillations intrinsic to the star are found. In the two bottom panels we used 15 nights from OSN in the $y$ filter and $b-y$ colour, respectively. The different properties of the time series are reflected in the amplitude spectra.

There are many frequencies present in $\epsilon$ Cep and their location can be identified in the WIRE spectrum. The periods range from $0.7-1.9 \mathrm{~h}$ and the amplitudes of highest peaks are in the range 1-3 $\mathrm{ppt}^{1}$, which is typical for low amplitude $\delta$ Scuti stars. The OSN amplitude spectra are more complicated to interpret, with two main regions of excess power around 12-17 and 24-28 c/day. This makes the extraction of closely spaced frequencies difficult and is a well-known problem for single-site

\footnotetext{
${ }^{1}$ We note that $1 \mathrm{ppt} \simeq 1.086 \mathrm{mmag}$.
}

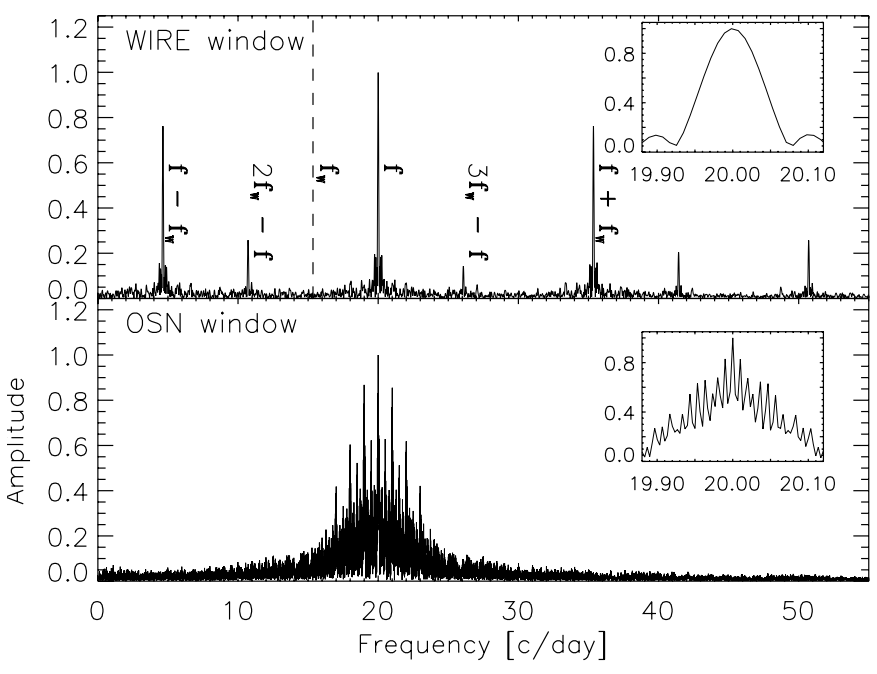

Fig. 3. Spectral windows for the WIRE (top panel) and osN data sets (bottom panel) computed for a single frequency at $f=20 \mathrm{c} / \mathrm{day}$. The insets show the details of the main peak.

observations of $\delta$ Scuti stars (Costa et al. 2003). For example, as a result of the combination of the frequencies $f_{2}$ to $f_{4}$, the highest peak in the OSN amplitude spectra is found at $\simeq 14 \mathrm{c} /$ day. Also, above $20 \mathrm{c} /$ day the highest peak in the OSN $y$ amplitude spectrum is found around $25.2 \mathrm{c} /$ day due to the combinations of $f_{1}$ and the close pair of frequencies $f_{5}$ and $f_{9}$.

In the following section we describe how we have extracted the individual frequencies from the light curves.

\section{Analysis of the $\epsilon$ Cep light curves}

\subsection{Using the superior WIRE spectral window}

Since the spectral window of the WIRE data set is less complicated than for the OSN data, we used the WIRE data to detect the significant frequencies. Also, the $S / N$ level is much higher in the WIRE data: in the cleaned amplitude spectrum the noise level is about 85 and $460 \mathrm{ppm}$ in the range 10-30 c/day for the WIRE and $y$ OSN data, respectively. In the OSN $v$ filter the noise level is $645 \mathrm{ppm}$ but the amplitudes are about $50 \%$ higher than in $y$. The WIRE data set is useful for avoiding the $1 \mathrm{c}$ /day aliasing problem that hamper our single-site ground-based data set and we can also detect additional frequencies with low amplitude.

We used the software package PERIOD04 by Lenz \& Breger (2005) for the extraction of the frequencies. After the extraction of the first frequency, the detection of additional frequencies is based on prewhitening or "cleaning" of the already detected frequencies. However, the solution is improved by a least-squares fit to the observations by a function of the form $\sum_{i=1}^{N} A_{i} \sin \left(2 \pi\left[f_{i} t+\phi_{i}\right]\right)$, thus each of the $N$ terms is determined by frequency $\left(f_{i}\right)$, phase $\left(\phi_{i}\right)$ and amplitude $\left(A_{i}\right)$. We note that the WIRE data set is very homogeneous and we therefore did not apply point weights.

The WIRE satellite observed $\epsilon$ Cep during $40 \%$ of its orbit, but the last part of each orbit is affected by scattered light which systematically offsets the measured flux. To minimize the effect of this we only used the part of the light curve which was unaffected by scattered light, i.e. this data set had a $30 \%$ duty cycle. From this data set we extracted 25 frequencies with $S / N$ above 4 . After subtracting these terms from the light curve we performed a decorrelation of the light curve with the background level and orbital phase. This allowed us to use the complete data set and 


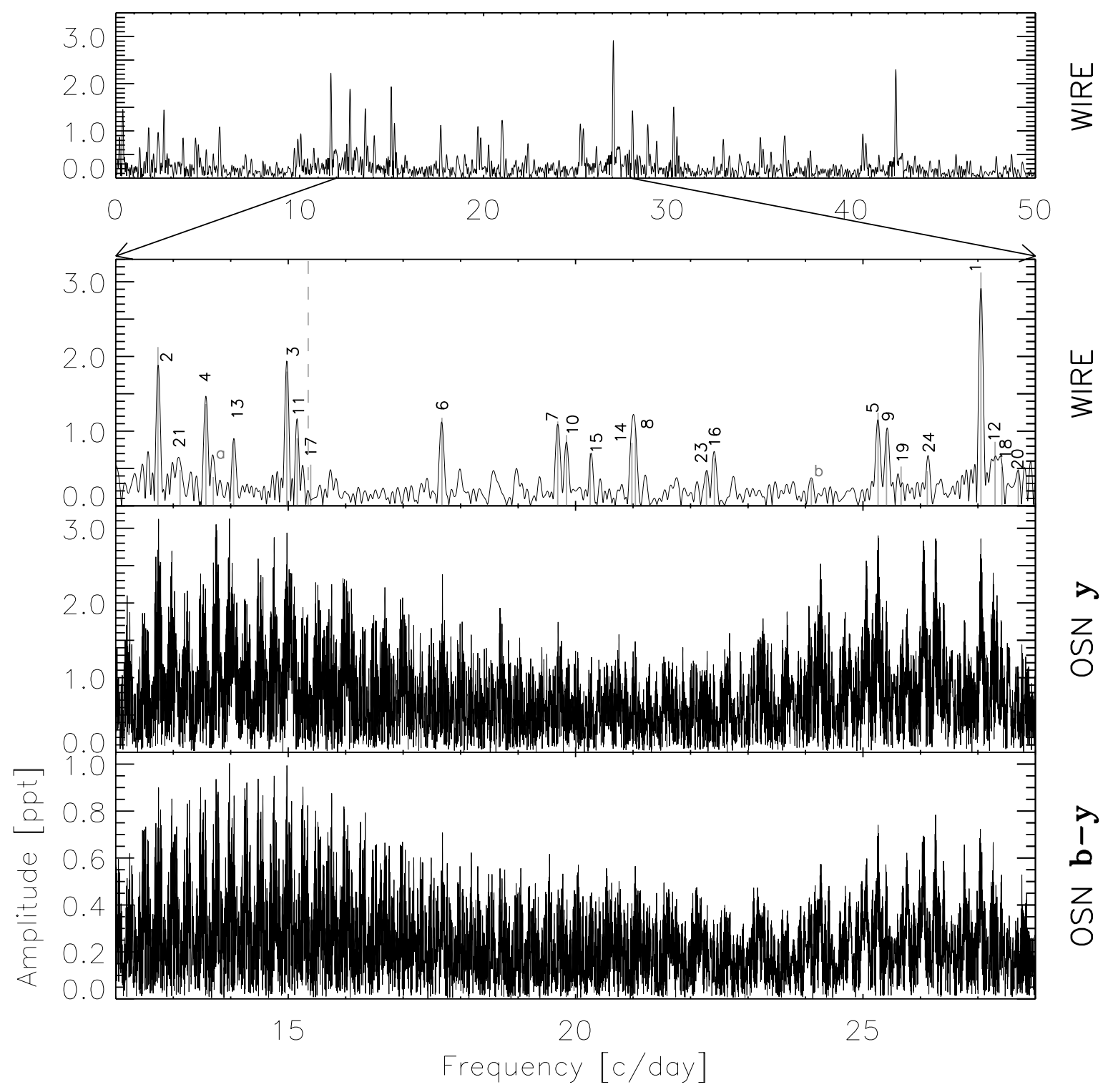

Fig. 4. The two top panels are the amplitude spectra of $\epsilon$ Cep based on the WIRE data set. The frequencies extracted from WIRE and the orbital frequency at $f_{\mathrm{W}}=15.348 \mathrm{c} /$ day (dashed line) have been marked. The peaks below $12 \mathrm{c} /$ day and above $28 \mathrm{c} /$ day are alias peaks due to the orbit of the WIRE satellite. The two bottom panels are the amplitude spectra of the OSN $y$ and $b-y$ data sets.

increase the duty cycle from $30 \%$ to $40 \%$. This greatly improves the spectral window and the first set of side lobes decrease from $88 \%$ to $76 \%$ while the second set of side lobes decrease from $58 \%$ to $25 \%$ (cf. top panel in Fig. 3).

Using the WIRE data set with $40 \%$ duty cycle we extracted 26 frequencies. The frequencies are marked in Fig. 4 and in Table 2 we list the frequency, amplitude, and phase of each frequency. Phases in Table 2 are given relative to the zero point in time, $t_{0}=2453175.5$. In the last column we give the $S / N$ which is the ratio of the amplitude and the noise level estimated in the cleaned amplitude spectrum. We will estimate the uncertainties on the frequency, phase, and amplitude based on simulations in Sect. 6.3. The first 24 frequencies in Table 2 have $S / N$ above 6 , and are numbered according to their $S / N$. The remaining two frequencies are less certain and are labeled $a$ and $b$.

We have searched for frequencies that are given as linear combinations of other terms within the frequency resolution, and for the WIRE data set this is $\delta f=3 / 2 T_{\text {obs }}=0.11 \mathrm{c} /$ day (Loumos $\&$ Deeming 1978). The three frequencies $f_{20}, f_{22}$, and $f_{a}$ are found to have low amplitude and found near these linear combinations: $f_{20}=f_{2}+f_{3}, f_{22}=f_{10}+f_{13}$, and $f_{a}=f_{12}-f_{4}=$ $f_{20}-f_{13}=f_{22}-f_{15}$. The many combinations for $f_{a}$ indicate that it is probably not intrinsic to the star. In addition, we find $f_{9}=2 \cdot f_{2}$ and $f_{18}=2 \cdot f_{a}$, which may be chance alignments.

\subsection{Analysis of the OSN light curves}

The OSN data set was collected 2.9 years prior to the WIRE data set. While some $\delta$ Scuti stars are known to have variable amplitudes we expect that the frequencies remain constant over such a short time scale. However, Breger \& Pamyatnykh (2006) found that the amplitude variation seen in FG Vir during several observing seasons can be explained by closely spaced frequencies. With this in mind we searched for frequencies using PERIOD04 while using the frequencies extracted from WIRE as a guide. We recovered the frequencies $f_{1}$ to $f_{9}$, but in some cases we had to apply an offset of the apparently highest peak by exactly $\pm 1 \mathrm{c} /$ day. We found evidence for $f_{11}, f_{12}$, and $f_{19}$ but these 
Table 2. Frequencies, amplitudes, phases, and $S / N$ for 26 individual frequencies extracted from the WIRE data set.

\begin{tabular}{rrrrr}
\hline \hline ID & $f_{i}[\mathrm{c} / \mathrm{day}]$ & $a_{i}[\mathrm{ppt}]$ & $\phi_{i}$ & $S / N$ \\
\hline$f_{1}$ & 27.053 & 3.12 & 0.994 & 41.2 \\
$f_{2}$ & 12.734 & 2.13 & 0.329 & 27.6 \\
$f_{3}$ & 14.976 & 1.80 & 0.524 & 23.4 \\
$f_{4}$ & 13.568 & 1.36 & 0.617 & 17.7 \\
$f_{5}$ & 25.262 & 1.25 & 0.096 & 16.1 \\
$f_{6}$ & 17.674 & 1.18 & 0.238 & 15.4 \\
$f_{7}$ & 19.689 & 1.13 & 0.858 & 14.6 \\
$f_{8}$ & 21.041 & 1.03 & 0.068 & 13.3 \\
$f_{9}$ & 25.409 & 0.99 & 0.541 & 12.8 \\
$f_{10}$ & 19.842 & 0.95 & 0.756 & 12.3 \\
$f_{11}$ & 15.157 & 0.90 & 0.939 & 11.8 \\
$f_{12}$ & 27.298 & 0.86 & 0.618 & 11.3 \\
$f_{13}$ & 14.050 & 0.86 & 0.573 & 11.2 \\
$f_{14}$ & 20.980 & 0.84 & 0.771 & 10.9 \\
$f_{15}$ & 20.255 & 0.70 & 0.781 & 9.1 \\
$f_{16}$ & 22.415 & 0.64 & 0.711 & 8.1 \\
$f_{17}$ & 15.392 & 0.55 & 0.489 & 7.2 \\
$f_{18}$ & 27.416 & 0.52 & 0.872 & 6.9 \\
$f_{19}$ & 25.663 & 0.53 & 0.013 & 6.8 \\
$f_{20}$ & 27.702 & 0.49 & 0.538 & 6.5 \\
$f_{21}$ & 13.118 & 0.48 & 0.131 & 6.3 \\
$f_{22}$ & 33.957 & 0.44 & 0.793 & 6.3 \\
$f_{23}$ & 22.277 & 0.48 & 0.101 & 6.1 \\
$f_{24}$ & 26.119 & 0.47 & 0.202 & 6.1 \\
$f_{a}^{1}$ & 13.697 & 0.39 & 0.486 & 5.0 \\
$f_{b}$ & 24.102 & 0.35 & 0.865 & 4.5 \\
$f_{10}+f_{13}$
\end{tabular}

${ }^{1}$ Note that $f_{a}=f_{12}-f_{4}=f_{20}-f_{13}=f_{22}-f_{15}$.

frequencies have low amplitude and the systematic offsets due to close neighbours and their aliases become significant.

Due to the long gaps in the OSN data sets there are aliases in the spectral window separated by $f_{\text {obs }} \simeq 1 / T_{\text {obs }}=0.009 \mathrm{c} /$ day. These aliases have almost equal amplitude but we can pick the right peak in the amplitude spectrum using the approximate frequency from the WIRE data set as we will demonstrate in Sect. 6.4. From the simulations in Sect. 6.3 we find the uncertainty on the WIRE frequencies to be $0.001-0.003 \mathrm{c} /$ day for the frequencies $f_{1}$ to $f_{6}$ and up to $0.004 \mathrm{c} /$ day for the frequencies $f_{7}$ to $f_{9}$. This means that the shift from one alias to the next in the OSN amplitude spectrum is at least at the 3-sigma level for $f_{1}$ to $f_{6}$ and about 2-sigma for $f_{7}$ to $f_{9}$.

Another method of cleaning the OSN data set is to assume that all frequencies with $S / N$ above 4 found in WIRE can be fitted to the OSN data set. In Fig. 5 we compare the frequencies and amplitudes found in the WIRE and OSN $y$ data sets. The top panel in Fig. 5 shows the ratio of amplitudes $a_{\mathrm{WIRE}} / a_{\mathrm{y}}$. The bottom panel shows the difference between the frequencies vs. the WIRE amplitude. The individual error bars are found from simulations. We find that the uncertainties are very large for the frequencies with low amplitude, but all frequencies agree within the uncertainties. That fact that several frequencies are found to have amplitudes that are different by more than $50 \%$ (marked by horizontal dashed lines in the top panel in Fig. 5) indicates that it is not sensible to fit all these frequencies to the oSN data, although they are present in the WIRE data set. The fact that we cannot extract all the frequencies found in the WIRE data set will systematically affect the parameters of the frequencies we extract from the OSN data sets. We have assessed this by doing simulations (see Sect. 6.3).

In Table 3 we summarize the frequency, amplitude, and phase of $f_{1}$ to $f_{8}$, which are clearly identified in the OSN data set.

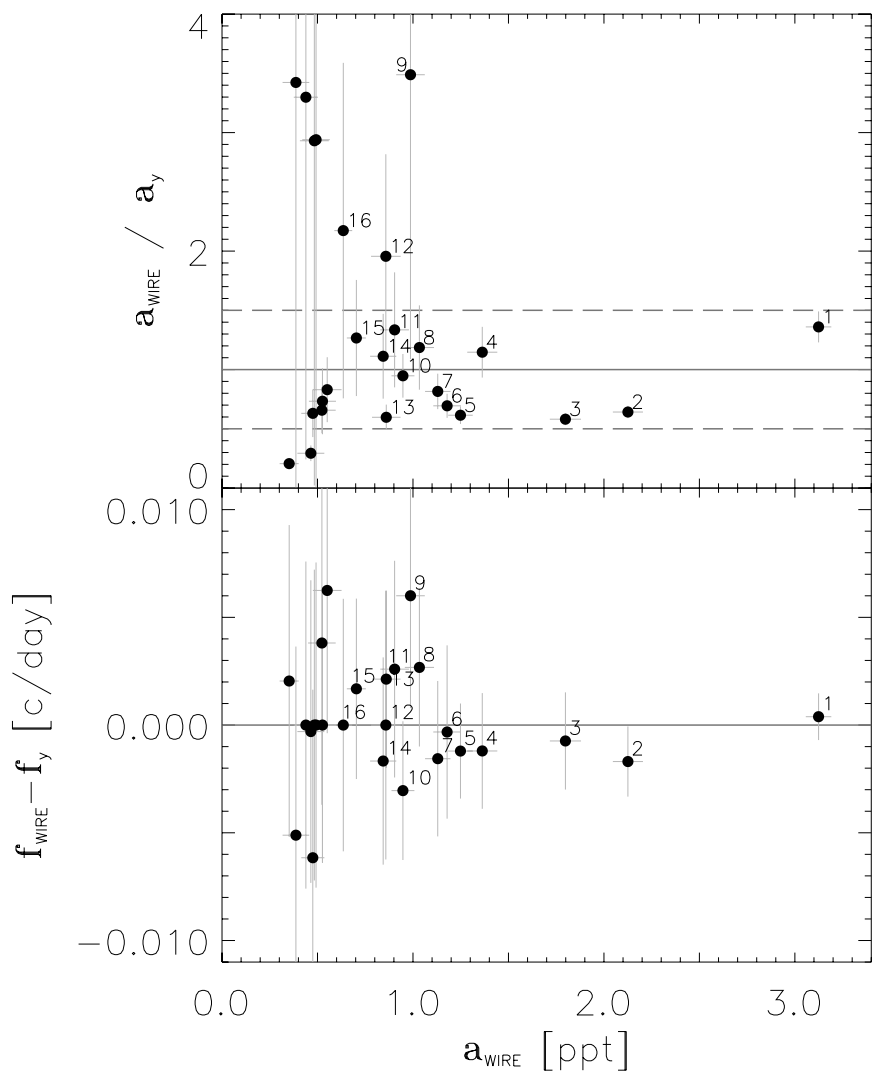

Fig. 5. We fitted all 26 frequencies found from the WIRE data set to the OSN $y$ data set. The panels show the ratio of the input and output amplitudes (top) and the difference between input and output frequencies.

The frequencies are the weighted mean values of the individual fits to uvby. The quoted error is the weighted mean error and is based on simulations done in Sect. 6.3. We give amplitudes in each of the four Strömgren filters and the colour light curve $b-y$. The phases of $y$ and $b-y$ are given relative to the zero point in time, $t_{0}=2452$ 130.0. Three of the frequencies, $f_{5}, f_{7}$, and $f_{8}$ have some closely spaced frequencies seen in the WIRE data set, namely $f_{9}, f_{10}$, and $f_{14}$. It is very likely that the parameters listed for these frequencies in Table 3 are systematically affected by this. We also note that $f_{7} \simeq f_{6}+2.0 \mathrm{c} /$ day and this will also affect the amplitude and phase.

\section{Accuracy of the extracted frequencies}

\subsection{Theoretical estimates}

The formal uncertainty on the frequency determined from a light curve is determined by the duration of the observing run, the number of data points, and $S / N$ ratio, i.e. the ratio of the amplitude to the noise in the light curve. The standard estimate of the uncertainty of the frequency is based on the least-squares covariance matrix or the Rayleigh resolution criterion. However, Schwarzenberg-Czerny (1991) demonstrated that both estimates are statistically incorrect. On one hand the least-squares covariance matrix does not account for correlation of residuals in the fit. Neglecting this may cause a large under-estimation of the uncertainty. On the other hand the Rayleigh resolution criterion is insensitive to the $S / N$ and therefore does not reflect the quality of the observations. 
Table 3. Frequencies and amplitudes in the Strömgren filters for frequencies extracted from the OSN data set. The frequencies are the weighted mean of the fit to each of the uvby light curves. Amplitudes are given for each filter in parts per thousand (ppt). Phases fitted to $y$ and $b-y$ are also given and indicated in units of the period. The three frequencies labeled $c l$ have a close neighbouring frequency with similar amplitude and the parameters are likely to be affected by this.

\begin{tabular}{l|r|rrrrrr|rr}
\hline \hline ID & $f_{\text {comb }}[\mathrm{c} / \mathrm{d}]$ & $a_{\mathrm{W}}$ & $a_{u}$ & $a_{v}$ & $a_{b}$ & $a_{y}$ & $a_{b-y}$ & $\phi_{y}$ & $\phi_{b-y}$ \\
\hline$f_{1}$ & $27.0522 \pm 0.0003$ & 3.12 & 2.76 & 3.12 & 2.61 & 2.13 & 0.496 & 0.570 & 0.584 \\
$f_{2}$ & $12.7357 \pm 0.0002$ & 2.13 & 4.15 & 5.15 & 3.78 & 3.19 & 0.713 & 0.046 & 0.130 \\
$f_{3}$ & $14.9766 \pm 0.0002$ & 1.80 & 3.78 & 4.61 & 3.79 & 2.82 & 1.085 & 0.447 & 0.470 \\
$f_{4}$ & $13.5688 \pm 0.0009$ & 1.36 & 1.80 & 2.12 & 1.64 & 1.17 & 0.459 & 0.373 & 0.462 \\
$f_{5}^{c l}$ & $25.2635 \pm 0.0004$ & 1.25 & 2.88 & 3.33 & 2.72 & 2.13 & 0.654 & 0.139 & 0.159 \\
$f_{6}$ & $17.6745 \pm 0.0004$ & 1.18 & 1.46 & 2.19 & 2.11 & 1.81 & 0.514 & 0.955 & 0.705 \\
$f_{7}^{c l}$ & $19.6904 \pm 0.0004$ & 1.13 & 1.27 & 2.00 & 1.89 & 1.34 & 0.639 & 0.315 & 0.106 \\
$f_{8}^{c l}$ & $21.0380 \pm 0.0004$ & 1.03 & 1.99 & 1.92 & 1.43 & 1.04 & 0.387 & 0.028 & 0.889 \\
\hline
\end{tabular}

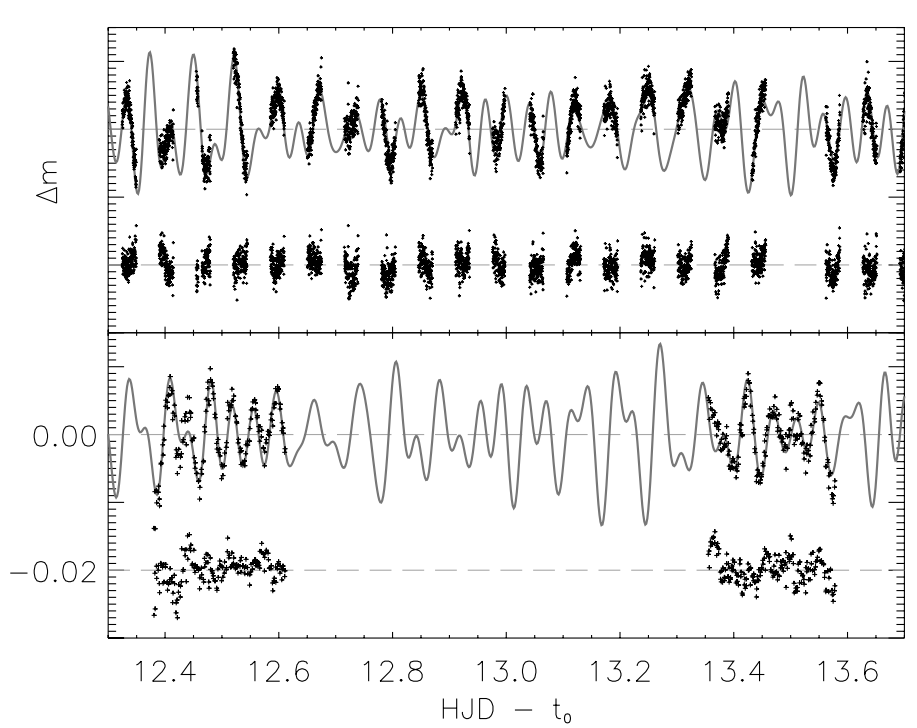

Fig. 6. Part of the light curves from WIRE (top panel) and OSN $y$ (bottom panel) including the fitted light curves in grey colour. The residuals are also shown but offset by -0.02 mag. As in Fig. 1 the zero-points $t_{0}$ are different.

For an ideal light curve with only white noise Montgomery \& O'Donoghue (1999) derived the uncertainty on the frequency, amplitude and phase (in radians) as

$\sigma\left(f_{i}\right)=\frac{\sqrt{6}}{\pi} \cdot \frac{1}{N^{1 / 2}} \cdot \frac{1}{T_{\mathrm{obs}}} \cdot \frac{\sigma}{a_{i}}$,

$\sigma\left(a_{i}\right)=\sqrt{\frac{2}{N}} \cdot \sigma$ and

$\sigma\left(\phi_{i}\right)=\sqrt{\frac{2}{N}} \cdot \frac{\sigma}{a_{i}}$

where $\sigma$ is the rms uncertainty per data point, $a_{i}$ is the amplitude, $N$ is the number of data points, and $T_{\text {obs }}$ is the observational time baseline. These uncertainties are strictly lower limits in the case of uncorrelated white noise. Using simple assumptions for correlated noise, Montgomery \& O'Donoghue (1999) found that the error estimates using the above equations are too optimistic by up to a factor of five. Following the suggestion by Montgomery \& O'Donoghue (1999) we calculated the correlation length $D$ from the autocorrelation of the cleaned amplitude spectra (after subtracting the mean) and located the intersection with zero. From the WIRE and OSN data sets we find

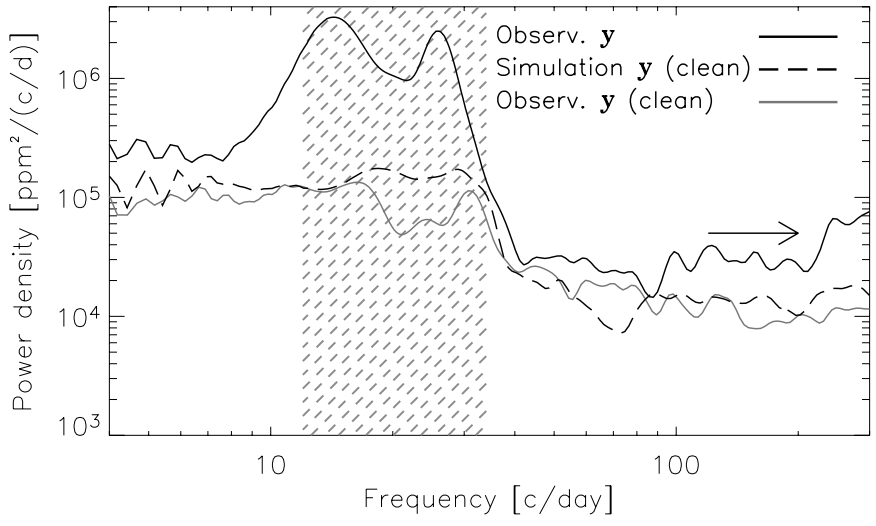

Fig. 7. Smoothed power density spectrum of the osN $y$ data set before and after cleaning (solid lines). The dashed line is for a simulation after cleaning. The location of the oscillations is marked by the hatched region.

$D_{\mathrm{WIRE}} \simeq 10.5, D_{\mathrm{OSN} ; y}=9.0$, and $D_{\mathrm{OSN} ; b-y}=10.5$, respectively, and we multiplied the error estimates from Eqs. (1)-(3) by the square root of the corresponding correlation length, $D$.

\subsection{Power density spectra}

For the simulations done in Sect. 6.3 we have found that it is important that the noise sources in the simulations mimic the observed data as closely as possible and we will investigate them in detail here.

In Fig. 6 we show part of the light curve of $\epsilon$ Cep from OSN $y$ and WIRE. The grey curves show the fit to each of the complete light curves. The residuals are also shown, offset by -0.02 mag. It is seen that there are significant systematic trends in the residuals which may be due to a combination of unresolved frequencies and instrumental drift.

The significance of this is seen more clearly in the frequency domain, and in Fig. 7 we show a smoothed version of the power density (PD) spectra of the OSN $y$ data set before and after the cleaning process. The region of excess power due to the oscillations is indicated by the hatched region (12-35 c/day). The PD is seen to increase by about an order of magnitude from the theoretical white noise level (>80 c/day). The slight increase in noise level above $200 \mathrm{c} /$ day (marked by the arrow) is because the telescope at OSN switched between the $\epsilon$ Cep and the comparison stars every 2-4 min.

A virtue of the PD spectrum is that we can compare the frequency dependence of the noise in different data sets even though the temporal coverage, time sampling, and number of 


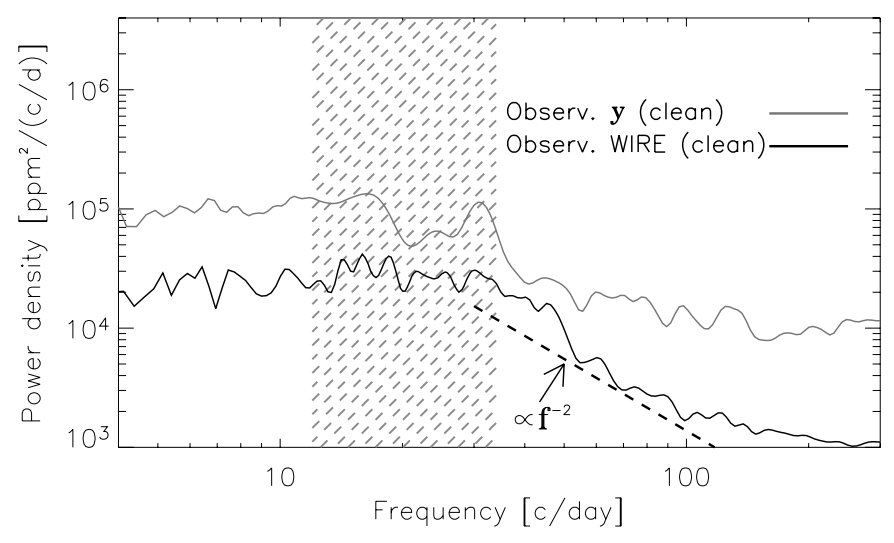

Fig. 8. Smoothed power density spectra of the cleaned OSN and WIRE data sets. The dashed line indicates the increase in noise towards low frequencies.

data points are quite different. In Fig. 8 we compare the PD spectra of the cleaned OSN $y$ (grey) and WIRE spectra (black solid line). The curves are similar in shape but there are fundamental differences. At the high frequency end the noise in OSN $y$ is higher by an order of magnitude and this is because the WIRE data set has $\sim 10$ times more data points than OSN and slightly lower uncertainty on each data point. At low frequencies ( $f<40 \mathrm{c} /$ day) the PD is still smaller for WIRE at all frequencies which means that there are additional noise sources present in the OSN data set. If the noise were intrinsic to the star the PD in WIRE and OSN would be identical at low frequencies. However, a large number of frequencies is present in $\epsilon$ Cep and a perfect cleaning of the OSN data set is not possible. This probably explains the higher PD at $f<40$ c/day compared to WIRE by a factor of $\simeq 3$.

The increase in the noise towards lower frequencies in the WIRE data set is likely due to a combination of instrumental drift and a number of undetected frequencies. This possibility was also discussed by Breger et al. (2005) in their analysis of the residuals after cleaning about 80 frequencies in the $\delta$ Scuti star FG Vir. We motioned in Sect. 1.1 that Kennelly et al. (1999) found several frequencies of high degree based on LPV studies. These frequencies will have negligible amplitude in photometry due to geometrical cancellation effects, but they may produce part of the increase in the noise that we observe.

\subsection{Simulations of the WIRE and OSN time series}

To confirm the theoretical error estimates in Sect. 6.1 and to better understand the different properties of the OSN and WIRE data sets we computed a large set of simulations. The OSN data set has long gaps of up to a month and thus the frequency analysis is hampered by a complicated spectral window. The interaction between frequencies can only be estimated by doing a large number of realistic simulations.

To mimic the large increase towards low frequencies discussed in Sect. 6.2, we added a number of frequencies with low amplitude in a wide frequency range. The simulations of the OSN $y$ filter and $b-y$ data set were done by including the 26 frequencies detected in WIRE but using the frequencies, amplitudes, and phases fitted to the observed data. We then added 110 frequencies with random (low) amplitudes from 0.0 to $0.4 \mathrm{ppt}$ and frequencies in the range 1-35 c/day for the $y$ filter. For the $b-y$ light curve we added 350 frequencies with amplitudes $0.0-0.1 \mathrm{ppt}$ in the same frequency range. Finally, we added a white noise component with rms of 1.85 and 1.60 mmag for $y$ and $b-y$, respectively. In the simulations of the WIRE data set we added 75 frequencies with random frequencies in the range 10$35 \mathrm{c} /$ day, random amplitudes in the range 0.0-0.3 ppt, random phases, and a white noise component with rms $1.7 \mathrm{mmag}$. An example of the cleaned PD spectrum of a simulation of an OSN $y$ time series is shown with a dashed line in the left panel in Fig. 7.

These ad hoc simulations roughly reproduce the increase in noise towards low frequencies while still no significant frequencies are present above the noise. We made 800 simulations of the OSN $y, b-y$, and WIRE light curves. We extracted frequencies from all simulations using an automated cleaning program using the procedure described in Sect. 5.1. For the OSN data set we also fitted the light curves when assuming the known frequencies and only fitting amplitudes and phases. We found the uncertainty by calculating the rms scatter in frequency, amplitude, and phase extracted from the 800 simulations.

In Fig. 9 we compare the uncertainties on frequency, amplitude, and phases as found from Eqs. (1)-(3) (solid lines) and the simulations (points). The left panel shows the uncertainties on the frequency, amplitude, and phase for OSN $y$ and the right panel is for OSN $b-y$, while the results for WIRE are shown with black points in both panels. The uncertainties of the simulations of the OSN data sets are shown when both frequency, amplitude and phase are fitted (grey $\times$ symbols) and the systematically lower uncertainties when only amplitude and phase are fitted (grey circle symbols). The improvement is indicated by a vertical arrow for each frequency. It can be seen that the frequencies are more accurately determined when using the osN $y$ data set. This is in agreement with Eq. (1) where the time baseline enters linearly while the number of data points enters as the square root; for the two data sets we approximately have the ratios $N_{\text {WIRE }} / N_{\text {OSN }} \simeq T_{\text {OSN }} / T_{\text {WIRE }} \simeq 10$, while the point-to-point noise, $\sigma$, is very similar. For the frequencies $f_{1}$ to $f_{8}$ the errors on frequency are about $0.001-0.003 \mathrm{c} /$ day in WIRE and $0.0005-$ $0.0010 \mathrm{c} /$ day in OSN. We should note that the OSN results rely on the important fact that we can select the right alias peak in the OSN data by using the approximate frequency found with WIRE; this is discussed in detail in Sect. 6.4. The uncertainties on amplitude and phase are independent of the time baseline and therefore they are more accurately determined from the WIRE data set since $N_{\mathrm{WIRE}} / N_{\mathrm{OSN}} \simeq 10$.

\subsection{Resolving the ambiguity of OSN alias peaks}

Due to long gaps in the OSN data set the spectral window has aliases of similar amplitude separated by $\Delta f \simeq 0.009 \mathrm{c} /$ day. If we only had the OSN data set, each frequency found in the observed data set can be offset by $n \times 0.009$ c/day for any $n= \pm 1, \pm 2$. However, using the frequencies found from WIRE we may choose the right alias sub-peak in the osN amplitude spectrum.

The results from our simulations in Fig. 10 illustrate that this is indeed possible. Each panel shows two histograms of the difference between the input frequency and the extracted frequency for $f_{1}$ to $f_{12}$ : the grey histogram is for the simulations of the WIRE data and black is used for OSN $y$. Several "groups" of frequencies separated by $0.009 \mathrm{c} /$ day are seen for the OSN simulations while a single but broader peak is seen for the distribution of extracted WIRE frequencies.

It can be seen that the uncertainty of the frequencies in the WIRE data set is sufficiently small that we can select the right peak in the OSN data set, at least for the dominant frequencies. We note that the uncertainties in the OSN simulations shown in 

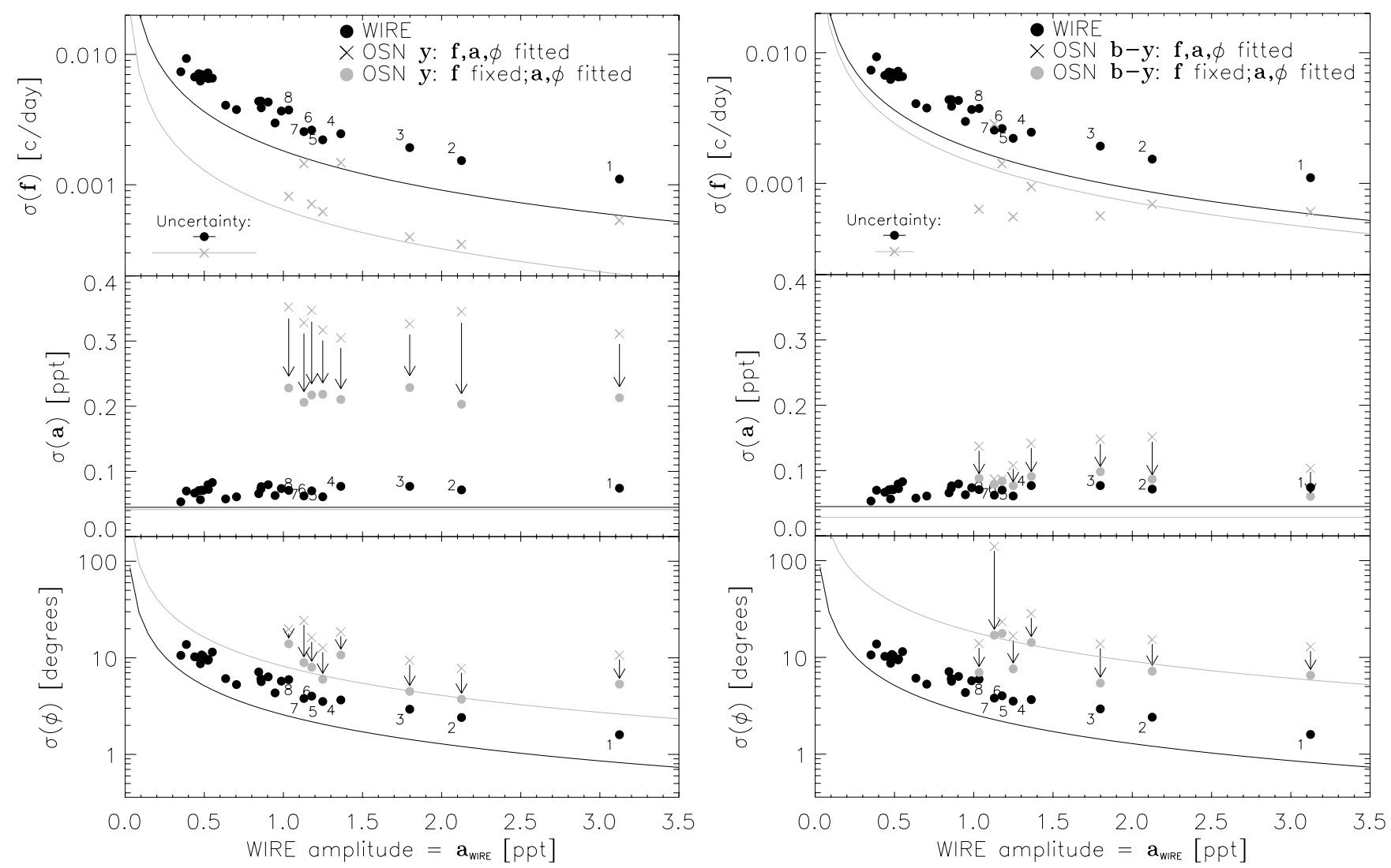

Fig. 9. Uncertainties on frequency, amplitude, and phase as found from simulations of WIRE (black) and osN (grey) data sets. The WIRE results are shown in both panels and the OSN results for $y$ and $b-y$ are shown in the left and right panels, respectively. When fixing the frequencies in the OSN data set the uncertainties on the amplitudes and phases decrease as indicated by arrows. The solid lines are the theoretical predictions for the uncertainty from Eqs. (1)-(3).

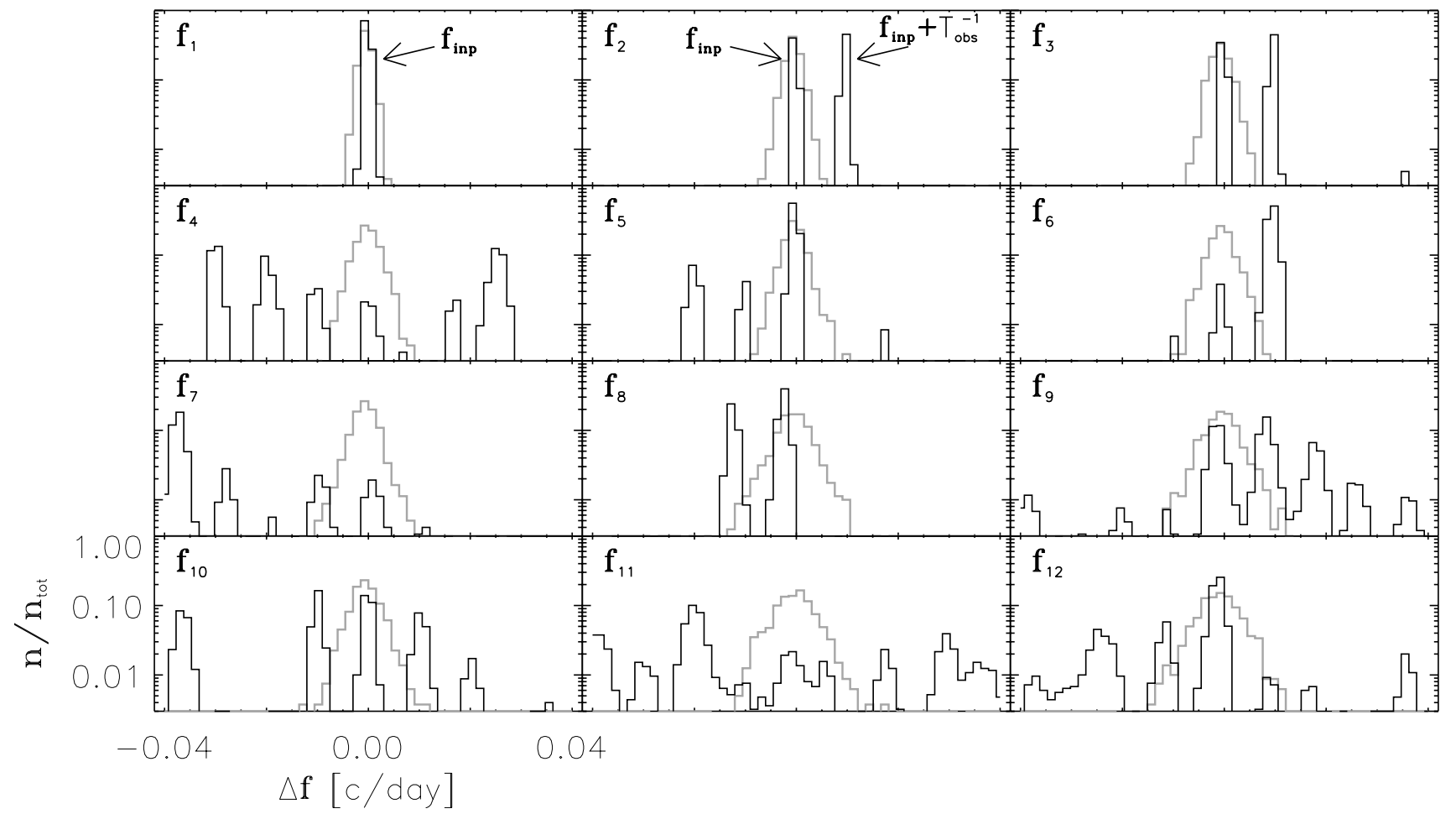

Fig. 10. Histogram of frequencies recovered in simulations of the OSN (black) and WIRE (grey) data sets for $f_{1}$ to $f_{12}$. In the panel for $f_{2}$ two groups of solutions in OSN separated by $1 / T_{\mathrm{obs}}=0.009 \mathrm{c} /$ day are marked. It is seen that the WIRE data set can be used to pick the right group, and that within this group the OSN data set has significantly lower uncertainty. 
Fig. 9 are based on the internal rms scatter within one "group" of extracted frequencies.

\section{Comparison of observations and models}

\subsection{Amplitude ratios and phase differences}

By measuring the parameters of frequencies in different filters we can infer the spherical degree, $l$, of the associated spherical harmonic. Each frequency can in principle be identified by measuring the phase difference and amplitude ratio in two filters as shown by Garrido et al. (1990) and Moya et al. (2004). In Fig. 11 we show the the amplitude ratio vs. the phase differences in the $y$ filter and $b-y$ colour for the three frequencies $f_{1}$ to $f_{3}$. The uncertainties are based on the simulations described in Sect. 6.3. The solid and dashed lines are results for a model with mass $M / M_{\odot}=1.65$ and $T_{\text {eff }}=7720 \mathrm{~K}$. We used an overshooting parameter of $d_{\mathrm{ov}}=0.2$ but did not include the effects of rotation. We note that in Sect. 3 we inferred a slightly cooler temperature, i.e. $T_{\text {eff }}=7340 \pm 150$ and an evolutionary mass of $M / M_{\odot}=1.75 \pm 0.20$. In the model shown in Fig. 11 the solid line is for $Q=0.033$ (fundamental radial mode, $n=1$ ) and the dashed line for $Q=0.017$ (third overtone, $n=4$ ) for a mixing length parameter $\alpha=0.5$. Amplitude ratios and phase differences were calculated for $l=0,1$, and 2: the highest amplitude ratio is for $l=2$ at $a_{b-y} / a_{y}>0.4$ and becomes progressively lower for decreasing $l$. The frequencies $f_{1}$ and $f_{2}$ are compatible with $l=1$ or 2 .

In general, the amplitude ratio and phase diagrams depend on the assumed mixing length parameter (Moya et al. 2004; Daszyńska-Daszkiewicz et al. 2003). Although the model used here does not describe $\epsilon$ Cep in detail, we can see that the observational uncertainties on the amplitude ratios and phases are too large to distinguish between the radial degree and overtone.

\subsection{Search for a pattern in the amplitude spectrum}

Regular frequency spacings similar to the large separation seen in solar-like stars have also been reported for some $\delta$ Scuti stars (Handler et al. 2000). Such a measurement would enable us to compare with theoretical predictions. We used the 24 most significant frequencies seen in $\epsilon$ Cep, namely those with $S / N$ above 6 to look for significant spacings using autocorrelation and histograms of frequency differences for different bin sizes.

In Fig. 12 we plot the power vs. the spacing frequency. We find a peak at $\Delta_{1}=2.41 \pm 0.02 \mathrm{c} / \mathrm{day}$. We did a series of simulations to see if the $\Delta_{1}$ spacing is indeed significant. Each simulation consist of 24 frequencies randomly distributed in the range 12-28 c/day. In many cases we found peaks in the amplitude spectrum with the same approximate location and amplitude as $\Delta_{1}$. Therefore we are extremely cautious about associating this with e.g. half of the large separation. The low number of observed frequencies as well as the narrow frequency range that is covered, are insufficient to consider $\Delta_{1}$ as being statistically significant.

We also used another technique to look for repetitive spacings among the frequencies by calculating frequency splitting histograms. Several frequency binning widths were used in order to search for recurring peaks. The only peak prevailing for all binning values is around $1.3 \mathrm{c} /$ day which roughly corresponds to the peak at $1.2 \mathrm{c} /$ day seen in Fig. 12. This spacing can be interpreted as the rotational splitting or perhaps the small separation. In the region of large differences, when varying the binning, two peaks are observed in the range of $4.8-5.0$ and $5.7-5.9 \mathrm{c} /$ day. We

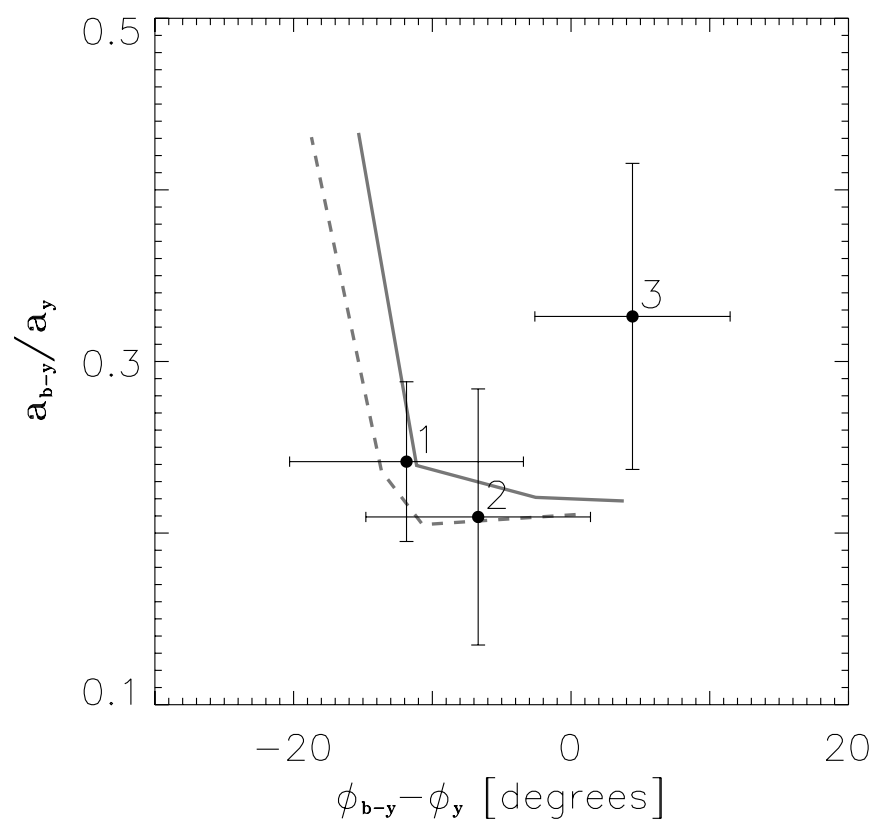

Fig. 11. Amplitude ratio and phase difference for the frequencies $f_{1}$ to $f_{3}$ measured in the OSN $y$ and $b-y$ light curves. The solid and dashed lines correspond to the fundamental mode and third overtone for a theoretical model with mass $M / M_{\odot}=1.65$.

are cautious about these large "candidate" splitting values, since we only observe frequencies in a narrow frequency range. The frequencies cover 12.7-34.0 c/day, so any spacing above half the width of this range cannot be considered: $\Delta f_{\text {lim }}=10.7 \mathrm{c} /$ day. Even at $5 \mathrm{c} /$ day we would only be able to detect an even spacing of four consecutive frequencies.

We computed a number of pulsation models within the photometric error box for $\epsilon$ Cep (cf. Fig. 2). We selected those models consistent with $\Delta_{1}$ being half the value of the mean large separation, i.e. $\Delta v \simeq 4.8 \mathrm{c} /$ day. The parameters of these models are given in Table 4. For each model we give the mass, radius, and luminosity in solar units, the effective temperature, the fractional hydrogen content in the central region, the large spacing of the frequencies, and the mean stellar density in solar units. We find that the observed peak in the histogram at $\simeq 1.3 \mathrm{c} /$ day is unlikely to be due to rotational splitting, since this peak is only seen in histograms of theoretical models for $m=0$ modes. Thus, if the reality of this spacing can be established from a more ambitious campaign, this splitting can only correspond to the small separation.

\section{Discussion and outlook}

We have analysed observations of the $\delta$ Scuti star $\epsilon$ Cep from the WIRE satellite and ground-based multi-colour uvby photometry from OSN. In some respects the WIRE data set is superior to OSN because the $S / N$ is a factor 5 higher and the spectral window is much more simple. Thus, we used the WIRE data set to extract the location of the oscillation frequencies. Due to the long gaps in the OSN light curve, the resulting spectral window is complicated. In addition to aliases at 1.0 and $0.5 \mathrm{c} /$ day, there are also "fine structure" sub-peaks with a spacing of $0.009 \mathrm{c} /$ day (roughly $\left.1 / T_{\text {obs }}\right)$. We performed a large set of realistic simulations to demonstrate that we can select the right sub-peak in the OSN amplitude spectrum, and in turn determine the frequencies of the dominant frequencies with accuracies of just $\simeq 0.0005 \mathrm{c} /$ day. The frequencies found with each of the four Strömgren filters agree 


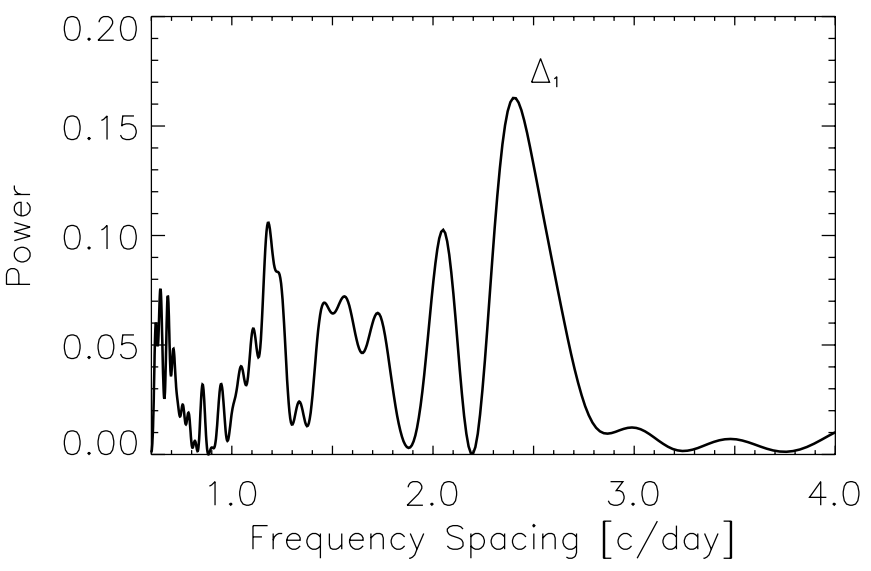

Fig. 12. The power spectrum used to search for frequency spacings in $\epsilon$ Cep. The 24 most significant frequencies found from the WIRE data set were used.

Table 4. Parameters of selected theoretical models for $\epsilon$ Cep. The mass, radius, and luminosity are given in solar units, $T_{\text {eff }}$ is the surface temperature, $X_{\mathrm{c}}$ is the central hydrogen fraction, $\Delta v$ is the large separation, and $\rho$ is the stellar mean density in solar units.

\begin{tabular}{ccccccc}
\hline \hline$M / M_{\odot}$ & $R / R_{\odot}$ & $L / L_{\odot}$ & $T_{\text {eff }}[\mathrm{K}]$ & $X_{\mathrm{c}}$ & $\Delta v_{l=0}\left[\mathrm{c} \mathrm{d}^{-1}\right]$ & $\rho / \rho_{\odot}$ \\
\hline 1.55 & 1.76 & 6.70 & 7000 & 0.48 & 4.99 & 0.283 \\
1.60 & 1.77 & 7.50 & 7190 & 0.50 & 4.94 & 0.287 \\
1.65 & 1.79 & 8.46 & 7370 & 0.51 & 4.95 & 0.288 \\
1.70 & 1.82 & 9.57 & 7530 & 0.51 & 4.89 & 0.281 \\
1.75 & 1.83 & 10.70 & 7720 & 0.52 & 4.90 & 0.284 \\
1.80 & 1.84 & 11.89 & 7910 & 0.53 & 4.92 & 0.288 \\
\hline
\end{tabular}

and we have computed the weighted mean frequency, thus improving in accuracy to $\simeq 0.0003 \mathrm{c} /$ day for the main frequencies (cf. Table 3).

Accurate frequencies are only of interest if they can be compared with theoretical models, and this requires that the degree of the mode can be determined. This can be done from the amplitude ratio and phase difference in different filters. We used the amplitudes and phases from the OSN $y$ filter and $b-y$ colour light curve, but our comparison with a theoretical model clearly indicates that the observed accuracies of phases and amplitude ratios are insufficient to perform a mode identification. While accurate frequencies can be obtained very effectively by extending the time baseline of the observations, accurate phases and amplitudes require higher $S / N$. The uncertainties we estimate from simulations tell us that around 25000 data points with $\simeq 2 \mathrm{mmag}$ point-to-point uncertainty are required, but we only have $10 \%$ of this available from OSN.

In order to use the method of the amplitude ratio vs. phase difference diagram we need a more complete monitoring of $\epsilon$ Cep. This would require a multi-site campaign with monitoring in two or more filters. This has been done for a number of $\delta$ Scuti stars where extensive multi-site campaigns with long temporal coverage were carried out. Examples are XX Pyx (Handler et al. 2000) and BI CMi (Breger et al. 2002). We propose to use highresolution time-series spectroscopy to study line-profile variations, which will make it possible to identify the modes. In addition, information on mode splitting (and azimuthal order $m$ ) can be achieved. When this is combined with additional photometric observations from ground (or space) we may hope to improve on the present work.

Perhaps the most interesting result from the current study is that we have detected several significant $(S / N=4.5-6.5)$ frequencies with very low amplitude: seven frequencies have amplitudes below 0.5 ppt. For many years is has been a puzzle why only some of the frequencies predicted from models of $\delta$ Scuti stars were in fact detected. Breger et al. (2005) discuss their results for FG Vir and point out that the "missing modes" are indeed there but that the detection level in previous studies was too poor. About two thirds of the frequencies detected in FG Vir have amplitudes in $y$ below 0.5 ppt. From the WIRE data of $\epsilon$ Cep we also find that several frequencies have amplitudes below $0.5 \mathrm{ppt}(\simeq y$ filter). The reason why we do not find even more frequencies with low amplitude is most likely that the frequency resolution in the data is too low. Even so, our results give support to the suggestion by Breger et al. (2005) that the modes predicted by models of $\delta$ Scuti stars are indeed present in the stars but one must have long temporal coverage with high photometric precision to be able to detect them.

The Canadian MOsT satellite (Walker et al. 2003) can monitor stars for up to 60 days with a duty cycle close to $100 \%$ with high photometric precision. Preliminary results for a $\delta$ Scuti star observed as a secondary target by MOST show about 80 frequencies to be present with amplitudes as low as $0.1 \mathrm{mmag}$ (J. M. Matthews, private communication). In the coming years two more dedicated photometry missions will be launched: COROT and KEPLER. The COROT mission (Baglin et al. 2001) will monitor several relatively bright stars for up to 150 days to obtain photometry with very high precision and with a duty cycle close to $100 \%$. However, observations are done in one filter only for all the missions mentioned here: WIRE, MOST, COROT, and KEPLER. Hence, ground-based support observations with multiple filters and/or spectroscopic measurements are needed in order to be able to identify the modes.

The present analysis of $\epsilon$ Cep has shown that much more complete and carefully planned ground-based observations are needed to avoid problems resulting from a complex spectral window. Also, it is necessary to collect enough multi-colour photometry to be able to measure phases and amplitudes with the required accuracy to be able to identify the modes. Multi-site campaigns that overlap in time with the space-based observations should be arranged for the future missions. For example, most primary COROT targets are quite bright $(V \simeq 6)$ and so long-term (i.e. months) monitoring with small $20-80 \mathrm{~cm}$ class telescopes with Strömgren filters and a stable photometer will be adequate to collect the necessary data. The missions have several secondary targets which are monitored at the same time as the primary target. Thus it will be a difficult but potentially valuable task to coordinate and collect all the necessary data.

Acknowledgements. H.B. is supported by the Danish Research Agency (Forskningsrådet for Natur og Univers), the Instrument center for Danish Astrophysics (IDA), and the Australian Research Council. H.B. is grateful to Torben Arentoft and Gerald Handler for useful discussions. J.C.S. acknowledges support from the Instituto de Astrofísica de Andalucía through an I3P contract financed by the European Social Fund and from the Spanish Plan Nacional del Espacio under project ESP2004-03855-C03-01.

\section{References}

Aerts, C., Handler, G., Arentoft, T., et al. 2002, MNRAS, 333, L35

Baade, D., Bardelli, S., Beaulieu, J. P., \& Vogel, S. 1993, A\&A, 269, 195

Baglin, A., Auvergne, M., Catala, C., Michel, E., \& COROT Team 2001, ESA SP-464: SOHO 10/GONG 2000 Workshop: Helio- and Asteroseismology at the Dawn of the Millennium, 10, 395

Bernacca, P.L., Perniotto, M. 1970, Contr. Oss. Astrof. Padova in Asiago, 239, 1 Bessell, M. S., Castelli, F., \& Plez, B. 1998, A\&A, 333, 231

Breger, M. 1966, ApJ, 146, 958

Garrido, R.; Handler, G.; Breger, M. et al. 2002, MNRAS, 329, 531 
Lenz, P., Antoci, V., Breger, M. et al. 2005, A\&A, 435, 955

Breger, M., \& Pamyatnykh, A. A. 2006, MNRAS, 339

Buzasi, D. L., Catanzarite, J., Laher, R. et al. 2000, ApJ, 532, 133

Buzasi, D. L., Bruntt, H., Bedding, T. R. et al. 2005, ApJ, 619, 1072

Bruntt, H., Kjeldsen, H., Buzasi, D.L., Bedding, T. R. 2005, ApJ, 633, 440

Bruntt, H., \& Buzasi, D. L. 2006, Mem. Soc. Astron. It., 77, 278

Costa, V., Rolland, A., López de Coca, P., Olivares, I., \& García-Pelayo, J. M. 2003, Asteroseismology Across the HR Diagram, 397

Daszyńska-Daszkiewicz J., Dziembowski W. A., Pamyatnykh A. A., 2003, A\&A, 407, 999

Erspamer, D., \& North, P. 2002, A\&A, 383, 227

Erspamer, D., \& North, P. 2003, A\&A, 398, 1121

ESA 1997, The Hipparcos and Tycho Catalogues, ESA SP-1200

Fesen, R. A. 1973, PASP, 85, 732

Garrido, R., Garcia-Lobo, E., \& Rodriguez, E. 1990, A\&A, 234, 262

Gray, D. F. 1971, PASP, 83, 103

Hacking, P., Lonsdale, C., Gautier, T. et al. 1999, eds. M. D. Bicay, R. M. Cutri, \& B. F. Madore, ASP Conf. Ser., 177, 409

Pikall, G.; O’Donoghue, D.; Handler, G. et al. 1997, MNRAS, 286, 303

Handler, G. et al. 2000, MNRAS, 318, 511

Hauck, B., \& Mermilliod, M., 1998, A\&AS, 129, 431

Kennelly, E. J., Brown, T. M., \& Ehrenfreund, P., 1999, ASPC, 185, 264
Kjeldsen, H., \& Frandsen, S. 1992, PASP, 104, 413

Kupka, F., \& Bruntt, H. 2001, First COROT/MOST/MOST Ground Support Workshop, 39

Lejeune, T., \& Schaerer, D. 2001, A\&A, 366, 538

Lenz, P., \& Breger, M. 2005, CoAst, 146, 53

Loumos, G. L., \& Deeming, T. J. 1978, Ap\&SS, 56, 285

Mantegazza, L., \& Poretti, E. 2002, A\&A, 396, 911

Mittermayer, P., \& Weiss, W. W. 2003, A\&A, 407, 1097

Montgomery, M.H., \& O’Donoghue, D. 1999, Delta Scuti Star Newsletter, 13, 28

Moya, A., Garrido, R., \& Dupret, M. A. 2004, A\&A, 414, 1081

Rodríguez, E., López-González, M. J., \& López de Coca, P. 2000, A\&AS, 144, 469

Rogers, N. Y. 1995, CoAst, 78

Royer, F., Grenier, S. Baylac, M.-O., Gómez, A. E., \& Zorec, J., 2002, A\&A, 393, 897

Schwarzenberg-Czerny, A. 1991, MNRAS, 253, 198

Suárez, J.C., Bruntt, H., \& Buzasi, D. 2005, A\&A, 438, 633

Walker, G., Matthews, J., \& Kusching, R. et al. 2003, PASP, 115, 1023

Zima, W. 1997, Delta Scuti Star Newsletter, 11, 37

Zima, W. et al. 2002, Radial and Non-radial Pulsations as Probes of Stellar Physics, IAU Colloq. 185, ASP Conf. Ser., 259, 598 